\title{
Tanimoto Based Similarity Measure for Intrusion Detection System
}

\author{
Alok Sharma, Sunil Pranit Lal \\ Faculty of Science, Technology and Environment, University of the South Pacific, Suva, Fiji \\ E-mail: "lal_s@usp.ac.fj \\ Received September 2, 2011; revised September 21, 2011; accepted October 4, 2011
}

\begin{abstract}
In this paper we introduced Tanimoto based similarity measure for host-based intrusions using binary feature set for training and classification. The k-nearest neighbor $(k N N)$ classifier has been utilized to classify a given process as either normal or attack. The experimentation is conducted on DARPA-1998 database for intrusion detection and compared with other existing techniques. The introduced similarity measure shows promising results by achieving less false positive rate at $100 \%$ detection rate.
\end{abstract}

Keywords: Intrusion Detection, $k$ NN Classifier, Similarity Measure, Anomaly Detection, Tanimoto Similarity Measure

\section{Introduction}

Intrusion detection is an important area in the field of computers and security, and in the recent years it has generated considerable interest in the research community. The intrusion detection system (IDS) can be subdivided into two main categories namely, signature-based detection and behavior-based detection. In this paper we focus on behavior-based detection which is also known as anomaly detection. An important feature of anomaly detection is that it can detect unknown attacks. Behavior modeling can be done by either modeling the user-behavior or process. The system call data is one of the most common types of data used for modeling process behavior. Host-based anomaly detection systems mostly focus on system call sequences with the assumption that a malicious activity results in an abnormal trace. Such data can be collected by logging the system calls using operating system utilities e.g. Linux strace or Solaris Basic Security Module (BSM). In this framework, it is assumed that the normal behavior can be profiled by a set of patterns of sequence of system calls. Any deviation from the normal pattern is termed as intrusion in this framework. An intrusion detection system needs to learn the normal behavior patterns from the previously collected data and this is normally accomplished by data mining or machine learning techniques. The problem of intrusion detection thus boils down to a supervised classification problem to identify anomalous sequences, which are measurably different from the normal behavior. The system call sequences of normal instances are used as the training set. Though anomaly-based IDS can detect unknown attacks, it suffers from having unacceptable false-positive rate [1]. This is because of the fact that it is hard to perfectly model a normal behavior. Unlike the traditional pattern recognition approach for classification, the aim in the present context is not only to achieve high accuracy rate but also to minimize the false positive rate. In recent years, a lot of research activities in anomaly detection focus on learning process behaviors and building the profiles with system call sequences as data sources.

Various machine learning techniques such as Support Vector Machines [2] and Neural Network [3] have been proposed for designing intelligent intrusion detection systems. Interested readers are directed to Tsai et al. [4] for a comprehensive overview on this subject. In this paper we use the $k \mathrm{NN}$ classification scheme [5-7] as an efficient means for intrusion detection. In carrying out the classification, it is a common practice to use features represented as frequency of system calls observed. While this approach has produced outstanding results [7], we are more interested in reducing the computational cost associated with classification task. Instead of representing features as frequency, which involves repetitive counting, we only consider absence or presence of a system call and represent it as a single bit of data. Needless to say binary representation consumes less storage space 
compared to integer representation. To this end, we propose a Tanimoto binary similarity measure, and empirically evaluate and compare its performance. To the best of authors' knowledge the result is better than other binary similarity schemes for intrusion detection reported in literature.

\section{A Brief Description of the Preceding Work}

In this section we briefly describe the research work on behavior-based intrusion detection procedures. Denning [8] did a pioneering work on behavior-based intrusion detection. In this approach profiles of subjects are learnt and statistical methods are used to compute deviations from the normal behavior. Lane and Brodly [9] propose another approach for capturing a user's behavior. A database of sequences of UNIX commands that normally a user issues, is maintained for each user. Any new command sequence is compared with this database using a similarity measure. Forrest et al. $[10,11]$ introduce a simple anomaly detection method based on monitoring the system calls invoked by active and privileged processes. The profile of normal behavior is built by enumerating all fixed length of unique and contiguous system calls that occur in the training data, and unmatched sequences in actual detection are considered abnormal. A similar approach is followed by Lee et al. [12], but they make use of a rule learner RIPPER, to form the rules for classification. Lee and Stolfo [13] use data mining approach to study a sample of system call data to characterize the sequences contained in normal data by a small set of rules. In monitoring and detection, the sequences violating those rules are treated as anomalies [14]. Warrender et al. [15] propose Hidden Markov Model (HMM) method for modeling and evaluating invisible events based on system calls. It is believed that the entire sequence of system calls in a process need not exhibit intrusive behavior, but few subsequences of very small lengths may possess the intrusive characteristics. Rawat et al [16] showed using rough set technique that the intrusive behavior in a process is very localized. Sharma et al. [7] introduce kernel based similarity measure for host-based intrusions. They have used $k \mathrm{NN}$ classifier to classify a process as either normal or abnormal.

Most of the IDSs that model the behavior of processes in terms of subsequences, take fixed-length, contiguous subsequences of system calls. One potential drawback of this approach is that the size of the database that contains fixed-length contiguous subsequences increases exponentially with the length of the subsequences. Wespi et al. [17] propose a variable length subsequence approach. Asaka et al. [18] develop another approach based on the discriminant method in which an optimal classification surface is first learned from samples of the properly labeled normal and abnormal system call sequences. Wang et al. [19] develop another Principle Component Analysis based method for anomaly intrusion detection with less computation efforts. Tandon and Chan [20] propose to consider system calls arguments and other parameters, along with the sequences of system calls. They make use of the variant of a rule learner LERAD (Learning Rules for Anomaly Detection).

In order to detect the deviation of anomalous system call sequences from the normal set of sequences, Liao and Vemuri [5] used a similarity measure based on the frequencies of system calls used by a program (process), rather than the temporal ordering. Their approach draws an analogy between text categorization and intrusion detection, such that each system call is treated as a word and a set of system calls generated by a process as a document. They used a 'bag of system calls' representation. Liao and Vemuri [5,21] adopted this representation to profile the behavior according to the trace of each process independently and a $k \mathrm{NN}$ method is used for classification. In this method, each system call is treated as a word and a collection of system calls during the execution of a process is treated as a document. The system call trace of a process is converted into a vector and cosine similarity measure is used to calculate the similarity among processes. In another study [22] by the same group, the Robust Support Vector Machine (RSVM) is applied to anomaly-based IDS. Recently, the emphasis of this RSVM study is on exhibiting the effectiveness of the method in the presence of noisy data. Rawat et al. [6] propose anomaly-based IDS. A new similarity measure called binary weighted cosine (BWC) is proposed and it is shown that by $k \mathrm{NN}$ classifier with the new measure, one can reduce the false positive rate substantially without sacrificing the detection rate. The authors have shown that by defining appropriate similarity measures, the detection by simple $k \mathrm{NN}$ can be as efficient as the sophisticated classification techniques like SVMs. Sharma et al. [7] propose a very efficient anomaly based IDS. They have introduced kernel based similarity measure and showed that it can capture similarity at very accurate level. They have used $k \mathrm{NN}$ as their classification scheme. The success of any such classification is hinged on two important aspects the similarity measure and the classification scheme.

\section{Notations and Descriptions}

In the remaining discussion $S=\left\{s_{1}, s_{2}, s_{3}, \cdots, s_{\mathrm{m}}\right\}$ denotes a set of unique system calls where $m=|S|$ is the number of system calls. The training set $X$ is defined 
as a set of labeled sequences $\left\{<Z_{i}, c_{i}>\mid Z_{i} \in S^{*} ; c_{i} \in\{0\right.$, $1\}$ where $Z_{i}$ is an input sequence of system calls or a process, $c_{i}$ is a corresponding class label denoting 0 for "normal" label and 1 for "intrusion" label and $S^{*}$ is the set of all finite strings of symbol of $S$. In this representation, the ordering information of adjacent system calls in the input sequence is not considered to be significant and only the frequency of each system call is preserved. Given the data set $X$, the goal of the learning algorithm is to find a classifier $h: S^{*} \rightarrow\{0,1\}$ that maximizes detection rate and minimizes false positive rate.

The vector-space model of Information Retrieval (IR) is also often used to represent the set of processes. A process is depicted as a binary vector to represent the occurrences of system call. The value 1 represents the occurrences of a system call in a process and its absence is represented by 0 . Thus we define $Z b_{i}$ the binary representation of $Z_{i}$, where the entries of $Z b_{i}$ is 1 , if the corresponding system call is present, and 0 , otherwise.

\section{Tanimoto Similarity Measure}

The concept of Tanimoto coefficient [23], is presented here for binary similarity. It is an extension of Jacquard coefficient, which is a binary similarity measure. Given two vectors of binary features, $Z b_{i}$ and, $Z b_{j}$ the binary Tanimoto coefficient is represented as

$$
\operatorname{BT}\left(Z_{i}, Z_{j}\right)=T\left(Z b_{i}, Z b_{j}\right)=\frac{Z b_{i} \cdot Z b_{j}}{\left\|Z b_{i}\right\|^{2}+\left\|Z b_{j}\right\|^{2}-Z b_{i} \cdot Z b_{j}}
$$

where $\|\bullet\|$ is the Euclidean norm.

The Jacquard and Tanimoto coefficients have been extensively applied in several fields ranging from studying the diversity of species in ecosystem [24], to measuring similarity between chemical compounds [25]. In this paper we experiment using Tanimoto coefficient to measure the similarity between processes represented as binary features.

\section{Binary Tanimoto Weighted Cosine (BTWC) Similarity Measure}

In order to define BTWC similarity measure, we first define cosine similarity measure [5]. The cosine similarity measure $\lambda\left(Z_{i}, Z_{j}\right)$ between any two processes $Z_{i}$ and $Z_{j}$ is defined as follows.

$$
\operatorname{Cos} \operatorname{Sim}\left(Z_{i}, Z_{j}\right)=\lambda\left(Z_{i}, Z_{j}\right)=\frac{Z_{i} \cdot Z_{j}}{\left\|Z_{i}\right\| \cdot\left\|Z_{j}\right\|}
$$

The motive behind multiplying binary Tanimoto and $\operatorname{CosSim}$ is that $\operatorname{Cos} \operatorname{Sim}\left(Z_{i}, Z_{j}\right)$ measures the similarity based on the frequency and binary Tanimoto is the weight associated with $Z_{i}$ and $Z_{j}$. In other words, binary Tanimoto tunes the similarity score $\operatorname{Cos} \operatorname{Sim}\left(Z_{i}, Z_{j}\right)$ according to the number of similar and dissimilar system calls between the two processes. The BTWC similarity measure can be given as

$$
\operatorname{BTWC}\left(Z_{i}, Z_{j}\right)=T\left(Z b_{i}, Z b_{j}\right) \times \operatorname{Cos} \operatorname{Sim}\left(Z_{i}, Z_{j}\right)
$$

Therefore, the similarity measure BTWC takes frequency and the number of common system calls into consideration while calculating similarity between two processes.

\section{K-Nearest Neighbors with the Similarity Measures}

The $k \mathrm{NN}$ classifier is a generalized form of NN classifier. In this approach the behavior of a new process is classified by collecting the majority of $k$ closest training processes. The average of these majority $k$ measures is computed which is compared with the threshold value to determine if the process is normal or attack. The pseudo code of $k \mathrm{NN}$ procedure with similarity measure is as follows.

Let the training set $X$ has $n$ processes such that $Z_{j} \in$ $X$. Let $P$ be any new process.

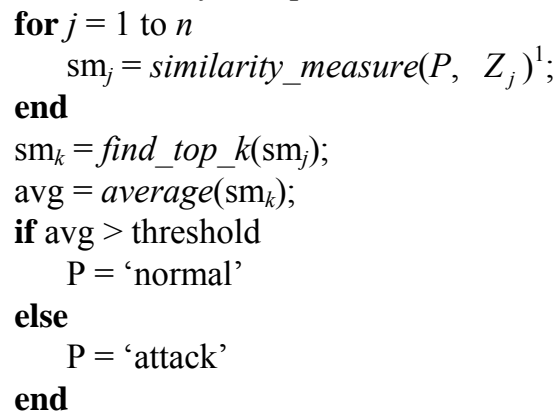

\section{An Illustration}

In this section, we analyze the proposed scheme with the help of an example. To illustrate, consider two training processes $Z_{1}$ and $Z_{2}$ associated with 10 unique system call $S$. Let also consider a test process $Z$ to measure the similarity with the training processes. The processes and the unique system call set $S$ are defined as follows:

$$
\begin{gathered}
S=\text { auditon, chdir, close, creat, kill, login, } \\
\text { mkdir, stat, su, sysinfo }\} \\
Z_{1}=\{\text { login, stat, stat, stat, stat, auditon, } \\
\text { auditon, auditon, auditon }\}
\end{gathered}
$$

\footnotetext{
${ }^{1}$ In place of similarity_measure, equation 1 or 3 will be used.
} 
$Z_{2}=\{\operatorname{login}$, close, su, sysinfo, stat, chdir, chdir, mkdir, creat, kill\}

$Z=\{$ login, auditon, auditon, stat, mkdir, close, close, creat, kill\}

To find the similarity between a test and train processes, we observe that there are only three common system calls between $Z$ and $Z_{1}$. However, there are six common system calls between $Z$ and $Z_{2}$. This inferred that there is more similarity between $Z$ and $Z_{2}$. Therefore, hypothetically $\operatorname{Sim}\left(Z, Z_{2}\right)>\operatorname{Sim}\left(Z, Z_{1}\right)$, where Sim is any similarity measure function. Computing the similarity score between these processes using CosSim, binary Tanimoto and BTWC similarity measures, we get,

$$
\begin{aligned}
\operatorname{Cos} \operatorname{Sim}\left(Z, Z_{1}\right)=0.6276 & \operatorname{Cos} \operatorname{Sim}\left(Z, Z_{2}\right)=0.5604 \\
\operatorname{BT}\left(Z, Z_{1}\right)=0.4286 & \operatorname{BT}\left(Z, Z_{2}\right)=0.6000 \\
\operatorname{BTWC}\left(Z, Z_{1}\right)=0.2690 & \operatorname{BTWC}\left(Z, Z_{2}\right)=0.3363
\end{aligned}
$$

According to CosSim similarity measure, $Z$ is more similar to $Z_{1}$ than to $Z_{2}$, since $\operatorname{Cos} \operatorname{Sim}\left(Z, Z_{1}\right)>$ $\operatorname{CosSim}\left(Z, Z_{2}\right)$. However, this contradicts with the hypothesis. On the other hand, using binary Tanimoto and BTWC similarity measures, $Z$ appeared to be more similar to $Z_{2}$ than to $Z_{1}$, since $\mathrm{BT}\left(Z, Z_{2}\right)>\mathrm{BT}\left(Z, Z_{1}\right)$ and $\operatorname{BTWC}\left(Z, Z_{2}\right)>\operatorname{BTWC}\left(Z, Z_{1}\right)$. The similarity scores measured by binary Tanimoto and BTWC validate the hypothesis. Therefore, it is more likely that by using these two techniques, better results for intrusion detection problem can be achieved.

In order to see the application of $k \mathrm{NN}$ classifier with binary Tanimoto and BTWC similarity measures, let us assume a third training process $Z_{3}$, such that $\mathrm{BT}(Z$, $\left.Z_{3}\right)=0.5$ and $\operatorname{BTWC}\left(Z, Z_{2}\right)=0.3$. Suppose the threshold for binary Tanimoto is $\theta_{\mathrm{BT}}=0.58$ and for BTWC is $\theta_{\text {BTWC }}=0.32$. If the classification procedure to label a process $Z$ into either as attack or normal is conducted by comparing the highest similarity score then by binary Tanimoto the process will be classified as normal since, $\mathrm{BT}\left(Z, Z_{2}\right)=0.6>\theta_{\mathrm{BT}}$ and by BTWC it will also be classified as normal since, $\operatorname{BTWC}\left(Z, Z_{2}\right)=0.3363>$ $\theta_{\text {BтwC }}$. This could give statistically unstable results as the classification is dominated by a single training process (with the highest similarity score). A better classification scheme would be to evaluate the average of top $k$ scores to arrive to the labeling of processes. If $k=2$ then for binary Tanimoto using $k \mathrm{NN}$ classifier the average of top 2 similarity scores would be $\operatorname{avg}<\theta_{\mathrm{BT}}$. This means that the process $Z$ is now classified as an attack since, avg $<\theta_{\mathrm{BT}}$. In a similar way, process $Z$ will be classified as an attack for BTWC using $k \mathrm{NN}$ classifier since, avg $=0.3181<\theta_{\mathrm{BTWC}}$. Therefore binary Tanimoto and BTWC similarity measures with $k \mathrm{NN}$ classifier are expected to give statistically stable results by comparing the average similarity measure of top $k$ processes.

\section{Experimentation}

In order to perform experimentation we use BSM audit logs from the 1998 DARPA data [26] for training and testing of our algorithm. This is the same data set used in previous research efforts [5-7] and thus it enables us compare the results. There are 50 unique system calls in the training data. All the 50 system calls are shown in Table 1.

In this dataset about 2000 normal sessions reported in the four days of data and the training data set consists of 606 unique processes. There are 412 normal sessions on the fifth day and we extract 5285 normal processes from these sessions. We use these 5285 normal processes as testing data. In order to test the detection capability of our method, we considered 54 intrusive sessions as test data. Table 2 lists these attacks. A number in the beginning of the name denotes the week and day followed by the name of the session (attack).

Table 1. List of 50 unique system calls.

access, audit, auditon, chdir, chmod, chown, close, creat, execve, exit, fchdir, fchown, fcntl, fork, fork1, getaudit, getmsg, ioctl, kill, link, login, logout, lstat, memcntl, mkdir, mmap, munmap, oldnice, oldsetgid, oldsetuid, oldutime, open, pathdonf, pipe, putmsg, readlink, rename, rmdir, setaudit, setegid, seteuid, setgroups, setpgrp, setrlimit, stat, statvfs, su, sysinfo, unlink, vfork

Table 2. List of 54 attacks used in test data.

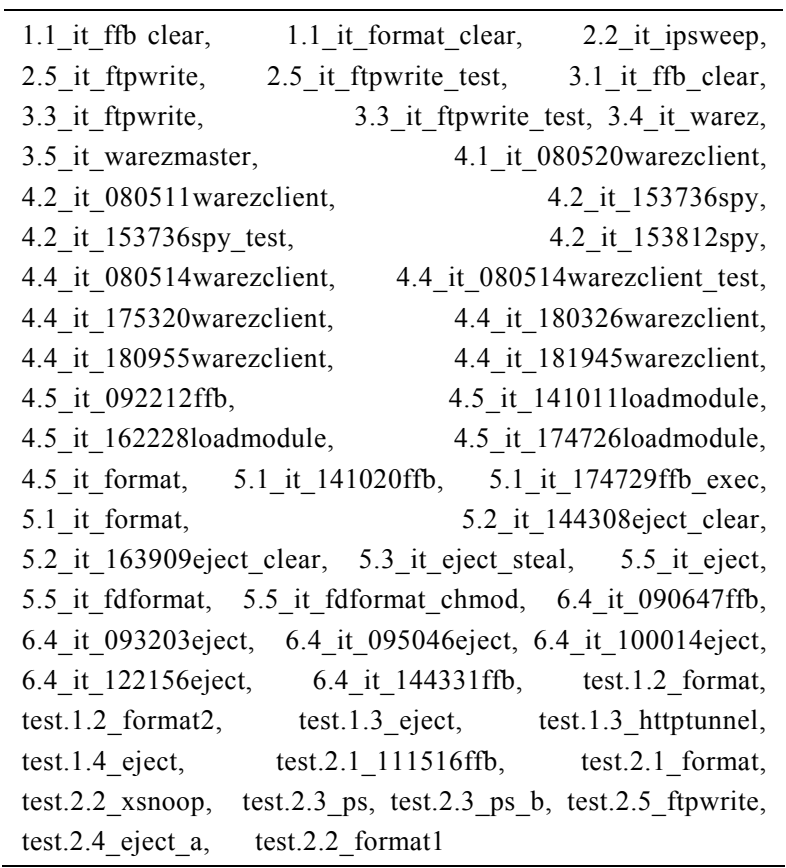


An intrusive session is said to be detected if any of the processes associated with this session is classified as abnormal. Thus detection rate is defined as the number of intrusive sessions detected, divided by the total number of intrusive sessions. We perform the experiments with $k=5$.

In Tables 3-6 we show Liao-Vemuri scheme [5], BWC scheme [6], BTWC scheme and binary Tanimoto scheme respectively for $k=5$. In the tables, the first column represents the threshold values used in the experiments. The second column depicts the false positives rates and the third column depicts the detection rate.

It can be seen from Table 3 that for Liao-Vemuri scheme the false positive rate is very high $(25.8 \%)$ at a detection rate of $100 \%$. It can be observed from Table 4 that BWC is a better technique than Liao-Vemuri as it provides lesser false positive rate $(4.65 \%)$ at a detection rate of $100 \%$. However, this false positive rate $(4.65 \%)$ still may not be acceptable. The BTWC scheme (Table 5) gives better performance by getting false positive rate of $4.1 \%$ at $100 \%$ detection. Next, the binary Tanimoto scheme performs the best by giving $3.7 \%$ false positive rate at a detection rate of $100 \%$. Table 7 summarizes the results obtained in Tables 3-6. It can be seen the binary

Table 3. Liao-vemuri scheme.

\begin{tabular}{ccc}
\hline Threshold & False Positive Rate & Detection Rate \\
\hline 0.52 & 0.0000 & 0.3519 \\
0.89 & 0.0009 & 0.7593 \\
0.99 & 0.0096 & 0.9630 \\
0.995 & 0.2575 & 1.0000 \\
\hline
\end{tabular}

Table 4. Binary weighted cosine scheme.

\begin{tabular}{ccc}
\hline Threshold & False Positive Rate & Detection Rate \\
\hline 0.52 & 0.0000 & 0.3704 \\
0.86 & 0.0095 & 0.9444 \\
0.90 & 0.0238 & 0.9815 \\
0.90099 & 0.0465 & 1.0000 \\
\hline
\end{tabular}

Table 5. BTWC scheme.

\begin{tabular}{ccc}
\hline Threshold & False Positive Rate & Detection Rate \\
\hline 0.52 & 0.0000 & 0.3704 \\
0.86 & 0.0348 & 0.9630 \\
0.88 & 0.0401 & 0.9630 \\
0.889 & 0.0411 & 1.0000 \\
\hline
\end{tabular}

Table 6. Binary tanimoto scheme.

\begin{tabular}{ccc}
\hline Threshold & False Positive Rate & Detection Rate \\
\hline 0.52 & 0.0000 & 0.3519 \\
0.86 & 0.0312 & 0.9630 \\
0.92 & 0.0369 & 0.9630 \\
0.9219 & 0.0369 & 1.0000 \\
\hline
\end{tabular}

Table 7. Summary: false positive rate at $100 \%$ detection rate for all the schemes.

\begin{tabular}{ccc}
\hline Method & False Positive Rate & Detection Rate \\
\hline Liao-Vemuri & $25.75 \%$ & $100.0 \%$ \\
BWC & $4.7 \%$ & $100.0 \%$ \\
BTWC & $4.1 \%$ & $100.0 \%$ \\
Binary Tanimoto & $3.7 \%$ & $100.0 \%$ \\
\hline
\end{tabular}

Tanimoto scheme is better than other schemes. The advantage of this scheme is that it utilizes only binary data which has lesser computing and managing requirements.

The receiver operating characteristic (ROC) curve is also depicted in Figure 1. It provides a comparison between the techniques. The ROC curve is a graph between the attack detection rate and false positive rate. It can be seen in the figure that binary Tanimoto converges to $100 \%$ detection rate faster than other three schemes. The curve of BTWC scheme is better than binary Tanimoto for smaller values of false positive rate. However, it converges slower to $100 \%$ detection rate than binary Tanimoto scheme. Nonetheless, the benchmark is to obtain lowest false positive rate at $100 \%$ detection rate and the proposed schemes are outperforming other similarity measures.

\section{Conclusions}

The Tanimoto based similarity measure schemes have been introduced in this work. It was observed that these schemes produced better results than other techniques. The best result obtained by Tanimoto scheme was 3.7\% at the detection rate of $100 \%$. To the best of the authors' knowledge the performance of the proposed technique is better than any previously documented result using binary similarity measure in the area of intrusion detection.

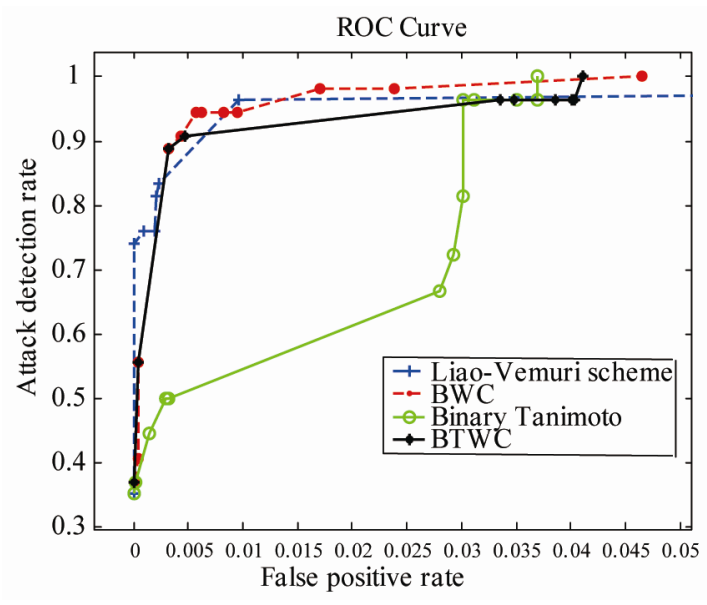

Figure 1. ROC curve for Liao-vemuri, BWC, binary tanimoto and BTWC schemes. 
It is worth highlighting that a binary representation of features captures less detailed information about a process compared with features represented as frequency. Even with this limitation, we obtained good results, which gives validity to our proposed approach.

\section{Acknowledgements}

The authors would like to thank the reviewers and the Editor for providing constructive comments about the paper.

\section{References}

[1] T. Lane and C. E. Brodley, "Temporal Sequence Learning and Data Reduction for Anomaly Detection," In Proceedings of 5th ACM Conference on Computer \& Communication Security, San Francisco, November 3-5, 1998, pp. 150-158.

[2] Y. Yi, J. Wu and W. Xu, "Incremental SVM Based on Reserved Set for Network Intrusion Detection," Expert Systems with Applications, Vol. 38, No. 6, 2011, pp. 76987707. doi:10.1016/j.eswa.2010.12.141

[3] G. Wang, J. Hao, J. Ma and L. Huang, “A New Approach to Intrusion Detection Using Artificial Neural Networks and Fuzzy Clustering," Expert Systems with Applications, Vol. 37, No. 9, 2010, pp. 6225-6232. doi:10.1016/j.eswa.2010.02.102

[4] C. F. Tsai, Y. F. Hsu, C. Y. Lin and W. Y. Lin, "Intrusion Detection by Machine Learning: A Review," Expert Systems with Applications, Vol. 36, No. 10, 2009, pp. 1199412000. doi:10.1016/j.eswa.2009.05.029

[5] Y. Liao and V. R. Vemuri, "Use of K-Nearest Neighbor Classifier for Intrusion Detection," Computers \& Security, Vol. 21, No. 5, 2002, pp. 439-448. doi:10.1016/S0167-4048(02)00514-X

[6] S. Rawat, V. P. Gulati, A. K. Pujari and V. R. Vemuri, "Intrusion Detection Using Text Processing Techniques with a Binary-Weighted Cosine Metric," Journal of Information Assurance and Security, Vol. 1, 2006, pp. 4350 .

[7] A. Sharma, A. K. Pujari and K. K. Paliwal, "Intrusion Detection Using Text Processing Techniques with a Kernel Based Similarity Measure," Computers \& Security, Vol. 26, No. 7-8, 2007, pp. 488-495. doi:10.1016/j.cose.2007.10.003

[8] D. E. Denning, “An Intrusion-Detection Model," In Proceedings of the 1986 IEEE Symposium on Security and Privacy (SSP '86), IEEE Computer Society Pressm, 1990, pp. 118-133.

[9] T. Lane and C. E. Brodly. "An Application of Machine Learning to Anomaly Detection," In Proceeding of the 20th National Information System Security Conference, Baltimore, MD, 1997, pp. 366-377.

[10] S. Forrest, S. A. Hofmeyr, A. Somayaji and T. A. Longstaff, "A Sense of Self for Unix Processes," Proceedings of the 1996 IEEE Symposium on Research in Security and Privacy, Los Alamos, 1996, pp.120-128.

[11] S. Forrest, S. A. Hofmeyr and A. Somayaji, "Computer Immunology," Communications of the ACM, Vol. 40, No. 10, 1997, pp. 88-96. doi:10.1145/262793.262811

[12] W. Lee, S. Stolfo and P. Chan. "Learning Patterns from Unix Process Execution Traces for Intrusion Detection," In Proceedings of the AAAI97 Workshop on AI Methods in Fraud and Risk Management, AAAI Press, Menlo Park, 1997, pp. 50-56.

[13] W. Lee and S. Stolfo, "Data Mining Approaches for Intrusion Detection," Proceedings of the 7th USENIX Security Symposium, Usenix Association, 1998, pp.79-94.

[14] E. Eskin, A. Arnold, M. Prerau, L. Portnoy and S. Stolfo, "A Geometric Framework for Unsupervised Anomaly Detection: Detecting Intrusions in Unlabeled Data," Applications of Data Mining in Computer Security, Kluwer Academics Publishers, Berlin, 2002, pp. 77-102.

[15] C. Warrender, S. Forrest and B. Pearlmutter, "Detecting Intrusions Using System Calls: Alternative Data Models," Proceedings of 1999 IEEE Symposium on Security and Privacy, Oakland, 1999, pp. 133-145.

[16] S. Rawat, V. P. Gulati and A. K. Pujari, "A Fast HostBased Intrusion Detection System Using Rough Set Theory," Computer Science, Vol. 3700, No. 2005, 2005, pp. 144-161. doi:10.1007/11574798 8

[17] A. Wespi, M. Dacier and H. Debar, "Intrusion Detection Using Variable-Length Audit Trail Patterns," Proceedings of the Third International Workshop on the Recent Advances in Intrusion Detection (RAID'2000), Toulouse, No. 1907, 2000.

[18] M. Asaka, T. Onabuta, T. Inove, S. Okazawa and S. Goto, "A New Intrusion Detection Method on Discriminant Analysis," IEICE Transaction on Information and Systems E84D, Vol. 5, 2001, pp. 570-577.

[19] W. Wang, X. Guan and X. Zhang, "A Novel Intrusion Detection Method Based on Principle Component Analysis in Computer Security," Proceedings of the International IEEE Symposium on Neural Networks, Dalian, Lecture Notes in Computer Science, Vol. 3174, No. 2004, 2004, pp. 657-662. doi:10.1007/978-3-540-28648-6_105

[20] G. Tandon and P. K. Chan, "Learning Useful System Call Attributes for Anomaly Detection," Proceedings of the 18th International Florida Artificial Intelligence Research Society (FLAIRS) Conference, Clearwater Beach, 2005, pp. 405-410.

[21] Y. Liao and V.R. Vemuri, "Using Text Categorization Techniques for Intrusion Detection," Proceedings USE NIX Security 2002, San Francisco, 2002, pp. 51-59.

[22] H. Wenjie, Y. Liao and V.R. Vemuri, "Robust Support Vector Machines for Anomaly Detection in Computer Security," In International Conference on Machine Learning, Los Angeles, 2003.

[23] T. T. Tanimoto, "IBM Internal Report $17^{\text {th }}$," November 1957. http://en.wikipedia.org/wiki/Jaccard index for details. 
[24] J. A. Wallwork, "The Distribution and Diversity of Soil Fauna," Academic Press, London. 1976.

[25] P. Willett, "Chemical Similarity Searching," Journal of Chemical Information and Computer Sciences, Vol. 38,
1998, pp. 983-996. doi:10.1021/ci9800211

[26] DARPA 1998 Data, "MIT Lincoln Laboratory," 2007. http://www.ll.mit.edu/IST/ideval/data/data index.html 